Advancements in Instrumentation

\title{
Development of a Novel and Rapid Fully Automated Genetic Testing System
}

\author{
Masayuki UeHARA*,** \\ *Core Technology Development Center, Corporate Research and Development Div., Seiko Epson Corporation, \\ 281 Fujimi, Suwa, Nagano 399-0293, Japan \\ **Department of Laboratory Medicine, Shinshu University Hospital, 3-1-1 Asahi, Matsumoto, Nagano 390-8621, \\ Japan
}

\begin{abstract}
We have developed a rapid genetic testing system integrating nucleic acid extraction, purification, amplification, and detection in a single cartridge. The system performs real-time polymerase chain reaction (PCR) after nucleic acid purification in a fully automated manner. RNase P, a housekeeping gene, was purified from human nasal epithelial cells using silica-coated magnetic beads and subjected to real-time PCR using a novel droplet-real-time-PCR machine. The process was completed within $13 \mathrm{~min}$. This system will be widely applicable for research and diagnostic uses.
\end{abstract}

Keywords Genetic testing system, nucleic acid purification, polymerase chain reaction, cartridge, droplet-real-time-PCR system, magnetic beads, human nasal epithelial cells

(Received June 30, 2016; Accepted August 2, 2016; Published December 10, 2016)

\section{Introduction}

Nucleic acid diagnostics are finding increasing applications in many fields, including infectious disease, cancer, and genetic testing. Polymerase chain reaction (PCR) is one of the most sensitive methods for detecting low concentrations of target genes and is a key method in all these fields. ${ }^{1}$ However, PCR is a relatively time-consuming and expensive method that requires much operator skill. It is desirable to improve PCR methods for high-throughput applications.

Sensitive rapid nucleic acid-based diagnostic systems are required in clinical practice for accurate and early diagnosis of diseases, particularly infectious diseases.,3 Commercially available PCR devices were essentially designed for centralized laboratories in hospitals and are used to analyze many samples simultaneously. However, manual labor and technical expertise are still necessary. The repetitive steps involved in PCR (nucleic acid extraction, centrifugation, liquid handling, purification, amplification, and detection) are time-consuming and laborious, and samples are easily contaminated during these processes. As current PCR systems are expensive, cheaper and compact PCR systems with simple handling are required for PCR-based testing to become standard rapid techniques in medical clinics.

Many researchers have studied the above problems, and many have reported PCR-based nucleic acid tests using microfluidic systems. $^{4-8}$ These studies have achieved nucleic acid extraction and amplification in approximately $60-225 \mathrm{~min}$. However, these processes are time-consuming, and a liquid transfer pump and valve or centrifugation is necessary.

Automated nucleic acid extraction and purification in a cartridge with reagents within several minutes would result in major savings of both time and labor in PCR processes. Although other methods are available for extracting DNA or

E-mail: mauehara@shinshu-u.ac.jp
RNA, we used a combination of superparamagnetic beads and magnetic force. ${ }^{9} \quad$ Several bioassays have also already demonstrated the utility of magnetic beads in microfluidics. ${ }^{10-13}$ Connection of such a cartridge extraction and purification system $^{9}$ to our novel droplet-real-time-PCR system ${ }^{14}$ allows PCR tests to be performed simply in high-throughput assays by simple pouring of the sample into the cartridge. This system can eliminate the problems associated with delivering materials such as nucleic acids, proteins, and cells that may adhere to sample tubes and allows liquid handling of all processes.

In this study, we developed an integrated system for cell lysis, nucleic acid extraction, purification (including washing and elution), reverse transcription (RT), PCR amplification, and detection using magnetic bead-based plug/droplet manipulation in a single cartridge. The system is small and simple and does not require external pumps and valves. Here we describe our automated genetic testing system in which all processes from loading of samples to real-time gene detection are performed in a cartridge within $13 \mathrm{~min}$. This system has potential utility for molecular analyses and associated diagnostic applications.

\section{Experimental}

\section{Cartridge construction}

The cartridge is a small container in which nucleic acids are eluted from magnetic beads and amplified by PCR. The cartridge is connected to a bottle, plunger, capillary, and PCR tube (Fig. 1a). Nucleic acids are extracted in the bottle and purified by passing through the capillary, which is $1.0 \mathrm{~mm}$ in diameter and shaped like a capillary tube, allowing liquid to flow in a longitudinal direction. The plunger, a movable part that functions like a syringe, delivers a predetermined amount of liquid from the downstream side of the capillary into the PCR tube, which accommodates the reaction solution during thermal cycling. 
(a)

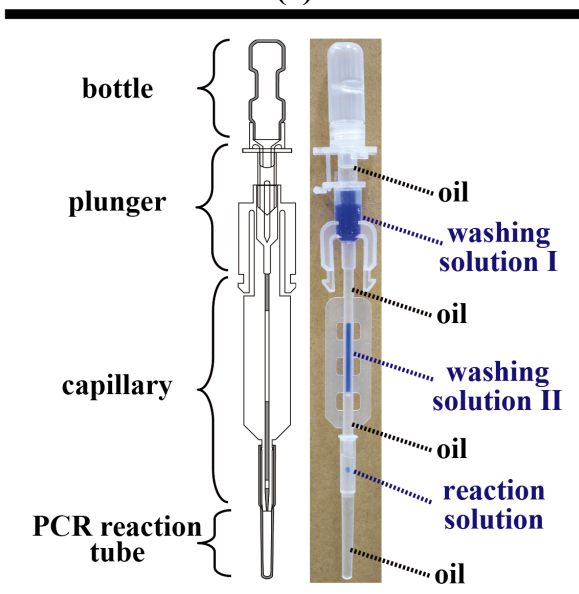

(b)

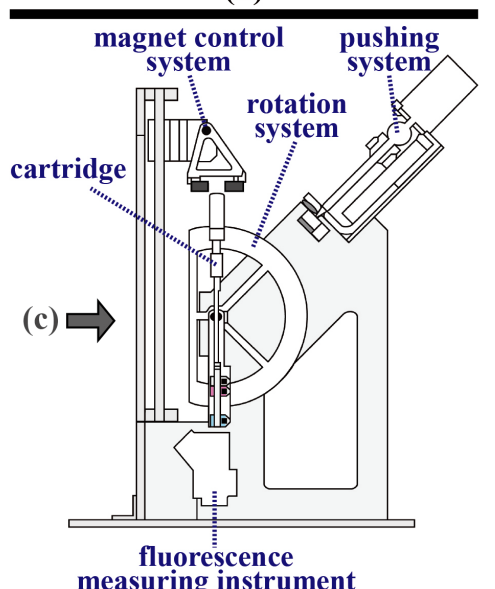

measuring instrument

(c)

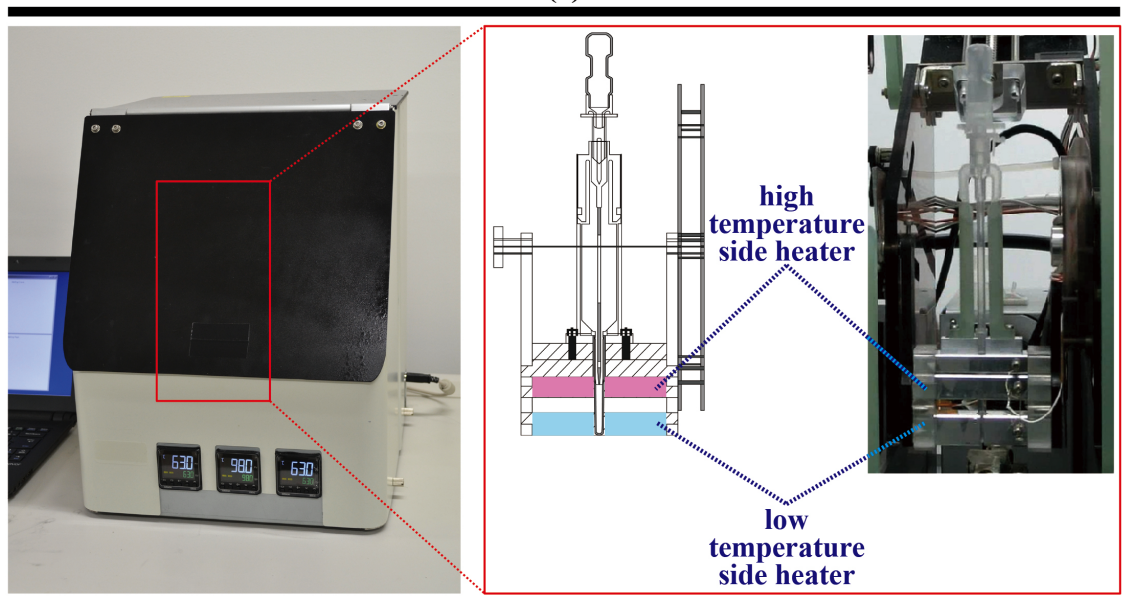

Fig. 1 Constituents of the cartridge and PCR device. (a) Internal constituents of the cartridge. The cartridge is composed of a bottle, a plunger, a capillary, and a PCR tube. (b) Internal constituents of the PCR device. Lateral view of the PCR device. The PCR device has a magnet control system, a pushing system, a fluorescence measurement instrument, and a rotation system. (c) Mounting portion of the rotary body. The cartridge is attached to the mounting portion of the rotary body. The mounting portion is equipped with heaters (a high-temperature heater on one side and a low-temperature heater on the opposite side).

The cartridge is equipped with an oil (2CS silicone oil) plug and plugs containing 280.0, 22.0, and $1.0 \mu \mathrm{L}$ of washing solution I, washing solution II, and elution (reaction) solution, respectively. The bottle contains $497.0 \mu \mathrm{L}$ of lysis and binding solution and $1.0 \mu \mathrm{L}$ of magnetic beads. The plunger is preloaded with oil and washing solution I.

\section{Device construction}

The PCR device has a magnet control system, a pushing system, an instrument for fluorescence detection, and a rotation system (Fig. 1b). Outer dimensions of the PCR device were $342 \times 390 \times 437 \mathrm{~mm}$ (Fig. 1c). The magnet control system controls magnetic beads in the cartridge with external magnets and has two magnetic components: one moves the beads up and down and the other causes them to vibrate (Fig. 2c). ${ }^{9}$ The pushing system controls the cartridge plunger and pushes the reaction solution plug and oil plug into the PCR tube (Figs. 3a, $3 \mathrm{~b}$, and $3 \mathrm{c}$ ). The rotation system rotates the cartridge and heater together (Fig. 1c). Gravity moves the droplet of PCR solution in the PCR tube between the two heaters with different temperatures. ${ }^{14}$ The thermal cycling process of PCR develops by rotation. The fluorescence measurement instrument measures the fluorescence of the reaction solution in the PCR tube.

\section{Operation}

The external magnets move the nucleic acids bound to the magnetic beads through the washing solution and transfer them into the reaction solution in the capillary. The pair of magnets sandwiches the cartridge at the given vertical portion and causes the magnetic beads to vibrate in the front-to-back direction (Fig. 2). We used a transport speed of $2.0 \mathrm{~mm} \mathrm{~s}^{-1}$ and a vibration frequency of $4.0 \mathrm{~Hz} .^{9}$

Nucleic acids bound to the magnetic beads are thus washed into the washing solution and eluted in the reaction solution (Fig. 2b). When the plunger is pushed into the capillary, the rod-like portion of the plunger fits into the lower syringe of the capillary (Figs. $3 \mathrm{~b}$ and $3 \mathrm{c}$ ). The droplet of reaction solution is then pushed out into the PCR tube.

The thermal cycling process is performed in the PCR tube attached to the cartridge (Figs. 3d, 3e, and 3f). The PCR tube contains oil, in which the reaction solution is isolated as a droplet (Fig. 3b). The specific gravity of the reaction solution is 


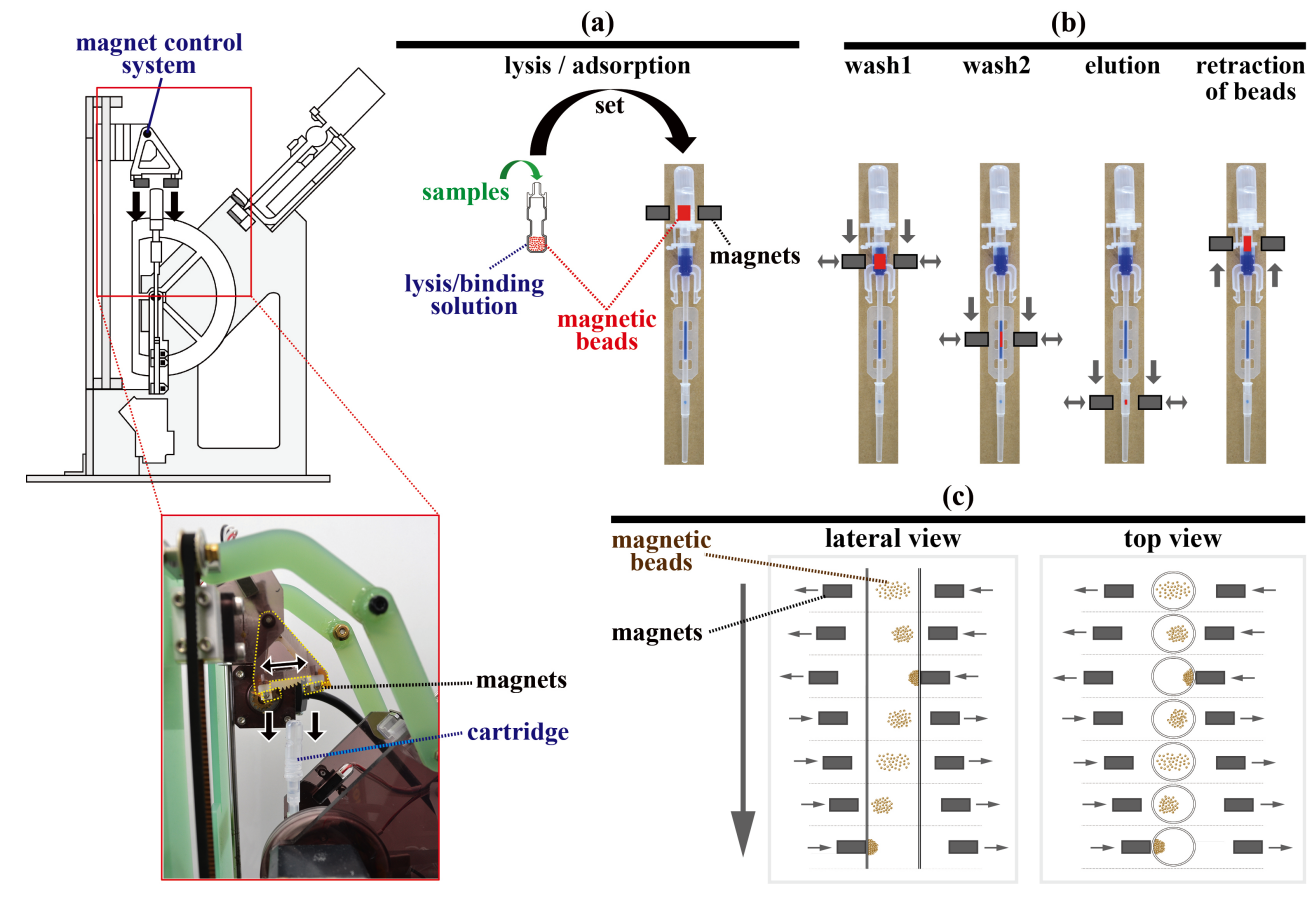

Fig. 2 Nucleic acid extraction and purification process. (a) The sample is poured into a bottle and the bottle is connected to the cartridge. (b) The outside magnets transfer nucleic acids bound to the magnetic beads into the reaction solution through washing I and II solutions. The outside magnets return to upper position. (c) Vertical movement of the outside magnets transfers magnetic beads vertically. The magnetic beads are vibrated by rapid horizontal movement of the exterior magnets.

greater than that of the oil, and the droplet readily moves to the bottom of the PCR tube. The droplet is thus transferred between high- and low-temperature sites under gravity by rotation of the PCR tube (Figs. 3e and 3f). ${ }^{14}$

\section{Fully automated system}

Using a magnet control system and plunger pushing system, our PCR system automates all nucleic acid test processes, including nucleic acid extraction, purification, amplification, and real-time detection (Figs. 2 and 3). The cartridge contains all assay reagents pre-packed in the plugs in unit-dose volumes separated by oil in the order in which they are used in a given assay. The PCR device has a magnet control system to move the magnetic beads along the cartridge. Accordingly, the movement of the magnetic beads, which are nucleic acidbinding solid-phase carriers, can be automated, and the magnetic beads can be moved in the same pattern each time. The PCR device also has a pushing system to push the plunger of the cartridge. Accordingly, the process for introducing the reaction solution plug, into which the nucleic acid has been eluted, into the PCR tube can be automated. An embedded microprocessor controls the actions of these processors to enable the system to perform all required assay procedures in the cartridge.

\section{System testing and validation}

RNase P, a housekeeping gene expressed in epithelial cells, was selected for evaluation of the system. Four samples containing $10,10^{2}, 10^{3}$, and $10^{4}$ human nasal epithelial cells (Takara Bio Co., Ltd. Japan) in $2.0 \mu \mathrm{L}$ of PBS were prepared. Aliquots of $2.0 \mu \mathrm{L}$ of each sample and $1.0 \mu \mathrm{L}$ of magnetic beads were added to the bottle with $497.0 \mu \mathrm{L}$ of lysis and binding solution (Fig. 2a). Nucleic acids were purified by transfer through the washing solutions of the Nucleic Acid
Purification MagExtractor $\mathrm{Kit}^{\mathrm{TM}}$ for Viral RNA (Toyobo Co., Ltd. Japan). The magnet control system vibrated the magnetic beads, moved them to the capillary of the cartridge, and performed all purification steps automatically (Figs. 2b and 2c).

RNase P was amplified in the PCR tube containing RNA purified from human nasal epithelial cells, SuperScript III/Platinum Taq Mix (Thermo Fisher Scientific, Waltham, MA), $5 \times$ reaction buffer (Tris- $\mathrm{HCl}, \mathrm{pH} 9.0, \mathrm{KCl}, \mathrm{MgCl}_{2}$ ), $1.2 \mu \mathrm{mol} / \mathrm{L}$ of each primer, $0.9 \mu \mathrm{mol} / \mathrm{L}$ of dual-labeled probe, and blocking solution in a total volume of $10.0 \mu \mathrm{L}$. Of the reaction mixture, $1.0 \mu \mathrm{L}$ was used for the reaction solution in the capillary. Primer and probe sequences were as follows: 5'-AGATTTGGACCTGCGAGCG-3'(forward), 5'-GAGCGGCTGTCTCCACAAGT-3' (reverse), and 5'-FAM-TTCTGACCTGAAGGCTCTGCGCG-BHQ1-3' (Sigma-Aldrich, Hokkaido, Japan). ${ }^{15}$ RT-PCR was performed according to the following profile: $63^{\circ} \mathrm{C}(30 \mathrm{~s})$ for $\mathrm{RT}$, followed by $98^{\circ} \mathrm{C}(10 \mathrm{~s})$, and 40 cycles of $98^{\circ} \mathrm{C}(4 \mathrm{~s})$ and $63^{\circ} \mathrm{C}(8 \mathrm{~s})$ for gene amplification and detection. As a negative control, samples without human nasal epithelial cells were also assayed. Confirmation of PCR products was performed by $4.0 \%$ agarose gel electrophoresis.

\section{Results and Discussion}

The RNase $\mathrm{P}$ gene was successfully amplified and detected from all samples containing $10-10^{4}$ nasal epithelial cells in $2.0 \mu \mathrm{L}$ of PBS using our system, and no amplification was observed in the negative control samples (Fig. 4a). The amplified PCR products in the droplet showed the expected size (65 bp) for RNase P, and no byproducts were observed (Fig. 4b). The entire process, including cell lysis, nucleic acid extraction, purification, amplification, and detection, took less than 13 min 


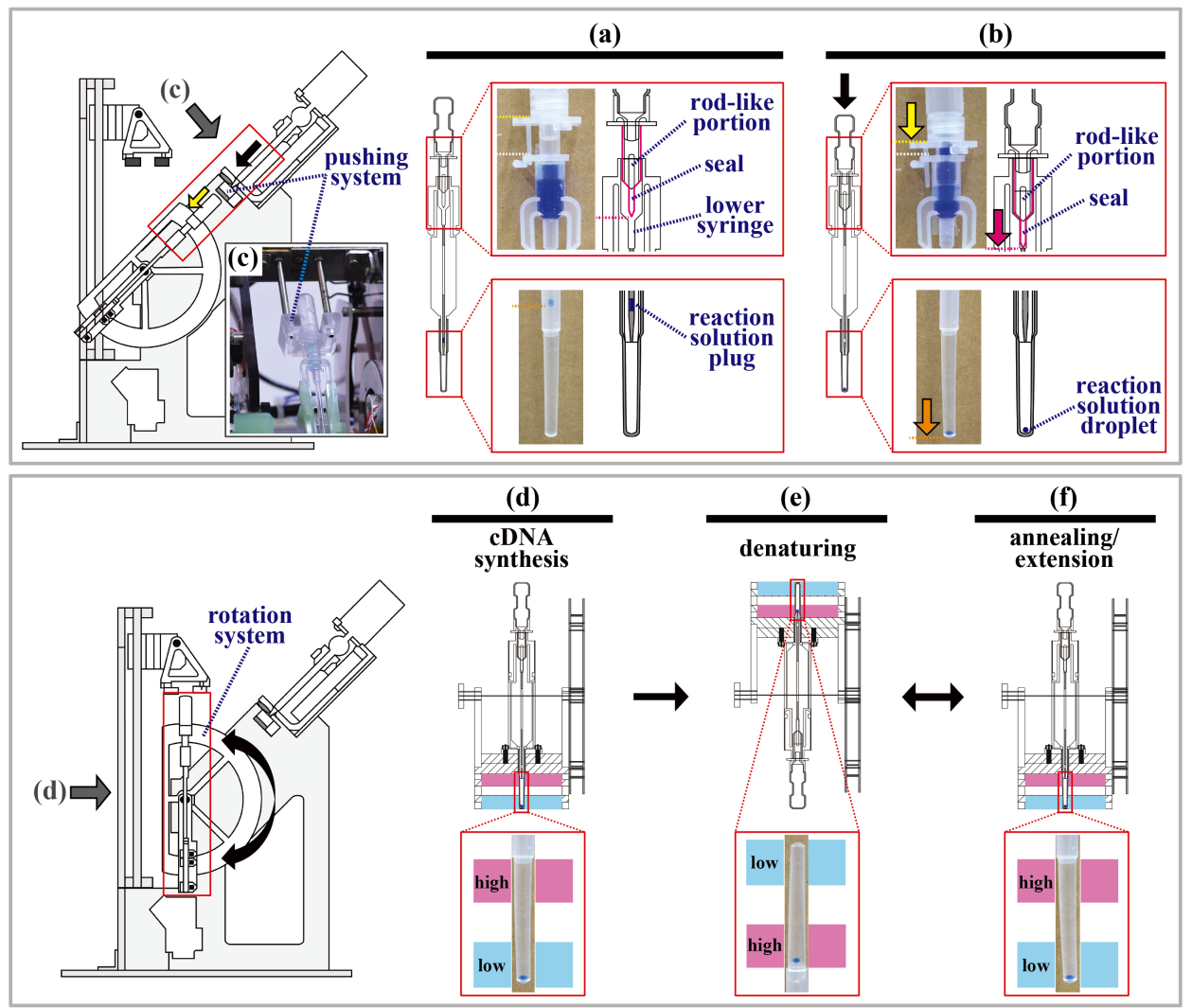

Fig. 3 Device operation. (a, b, c) Solution droplet formation process. (a) A plug in the capillary when the magnets have returned to the plunger portion. $(b, c)$ Droplet at the bottom of the reaction tube after the plunger has been pushed. When the plunger is pushed into the capillary, the rod-like portion of the plunger fits into the lower syringe of the capillary. (d, e, f) Thermal cycling process. (d, f) Droplet in the portion of the reaction tube controlled at a given low temperature. (e) Droplet in the portion of the reaction tube controlled at a given high temperature. The cartridge is flipped vertically with thermal cycling, and the PCR solution droplet moves alternately between the low- and hightemperature sides.

(a)

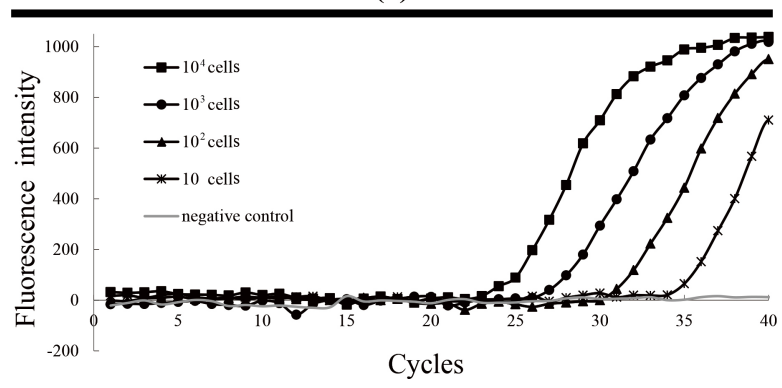

(b)

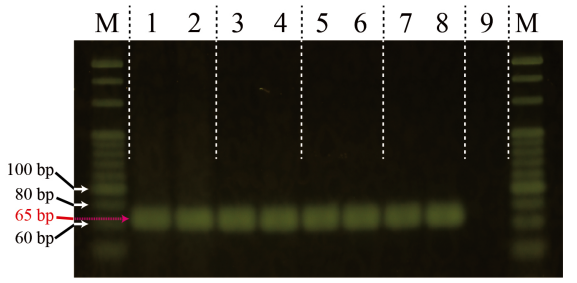

Fig. 4 Fluorescence curves and confirmation of amplified PCR products. (a) Real-time fluorescence curves of RNase P in total RNA samples purified from human nasal epithelial cells. Black lines represent RNase P curves: $\times$ 10-epithelial cells, $\boldsymbol{\Delta} 10^{2}$-epithelial cells, $\bullet 10^{3}$-epithelial cells, and $\boldsymbol{\square}$ $10^{4}$-epithelial cells. Gray line shows negative control without epithelial cells. (b) Agarose gel electrophoresis confirming amplified PCR products (65 bp). Lanes 1-2: $10^{4}$ epithelial cell samples; lanes $3-4: 10^{3}$ epithelial cell samples; lanes 5-6: $10^{2}$ epithelial cell samples; lanes 7 - 8: 10 epithelial cell samples; lane 9: negative control without epithelial cell samples; M: size marker.

to yield the results.

Our rapid and fully automated PCR system yielded PCR results in less than $13 \mathrm{~min}$ after the addition of raw samples to the cartridge. The entire process, including nucleic acid extraction, purification, amplification, and detection, could be completed in a single cartridge in an automated manner, and no specific training for gene handling was required. Our new PCR system will make it possible to perform rapid PCR tests in outpatient clinics in addition to relatively large hospitals. 


\section{Rapid detection system}

The reaction time of our PCR system using two novel technologies, a magnet control system and a droplet-real-timePCR system, was reduced to $13 \mathrm{~min}$, whereas that of conventional PCR systems is about $110 \mathrm{~min}$. First, aggregation and diffusion of the magnetic beads inside the capillary is regulated, washing and elution processes are enhanced, and total purification time is shortened. With our system, the purification (including cell lysis) time is reduced to within $3 \mathrm{~min}$, whereas the standard protocol takes about $30 \mathrm{~min}$. Second, the system reduces the RT-PCR time; the RT-PCR module detected RNase $\mathrm{P}$ within $10 \mathrm{~min}$ in comparison with about $80 \mathrm{~min}$ for conventional real-time RT-PCR. Because the system has two heating blocks set at different optimal temperatures and the reaction mixture moves under the force of gravity between these two regions during rotation of the PCR tube, no extra time is required to change the temperature of the reaction mixture, as is required by conventional real-time PCR machines with only one thermal incubator. This feature of the droplet-real-time-PCR module enables reactions to proceed at high rates. Reaction conditions, including thermal conditions and reagent concentrations, can also be modified to shorten the process.

\section{Other advantages}

In our PCR system, the RT-PCR reagent solution is injected as a short plug in a small volume (Figs. 1 and 3). Only $1.0 \mu \mathrm{L}$ of RT-PCR reagent solution is used, in contrast to the $20.0-50.0$ $\mu \mathrm{L}$ used in a conventional PCR system, resulting in reduced reagent costs. The system design also has other advantages. Positioning the rotary shaft closer to the capillary than to the PCR reaction tube allows the device to be miniaturized (Fig. 1c). The risk of cross-contamination is minimized because individual assays are compartmentalized in aqueous plugs separated by immiscible oil (Fig. 1a).

The system has various potential applications. Biological material used for extraction, purification, and amplification of nucleic acids is not limited to cells and may include viruses. Cells can be procured from any source, including microorganisms, tissue fragments, and blood. This innate flexibility will allow our system to be used in many different fields of science.

\section{Conclusions}

We have developed a rapid, fully automated genetic testing system for automated nucleic acid extraction, purification, amplification, and gene detection that produced results from raw samples within $13 \mathrm{~min}$. The on-cartridge system was validated using concentrations of $10-10^{4}$ human nasal epithelial cells, and real-time RT-PCR detection of the RNase P gene was achieved with as few as 10 cells. The system has the potential to improve genetic testing in several fields of science, including medical diagnostics.

\section{Acknowledgements}

I thank T. Honda and F. Takagi for critical comments, as well as H. Koeda, T. Murayama, Y. Saito, M. Hanamura, K. Yamada, K. Idegami, R. Kanai, S. Koide, T. Negishi, and T. Arai for technical assistance. This work was supported by grants from the Ministry of Education, Culture, Sports, Science and Technology (MEXT) Japan.

\section{References}

1. C. A. Heid, J. Stevens, K. J. Livak, and P. M. Williams, Genome Res., 1996, 6, 986.

2. I. M. Mackay, K. E. Arden, and A. Nitsche, Nucleic Acids Res., 2002, 30, 1292.

3. R. M. Ratcliff, G. Chang, T. Kok, and T. P. Sloots, Curr. Issues Mol. Biol., 2007, 2, 87.

4. G. Czilwik, T. Messinger, O. Strohmeier, S. Wadle, F. Stetten, N. Paust, G. Roth, R. Zengerle, P. Saarinen, J. Niittymäki, K. McAllister, O. Sheils, J. O'Leary, and D. Mark, Lab Chip, 2015, 15, 3749.

5. B. Zhuang, J. Han, G. Xiang, W. Gan, S. Wang, D. Wang, L. Wang, J. Sun, C.X. Li, and P. Liu, Lab Chip, 2015, 16, 86.

6. S. D. Goldenberg and J. D. Edgeworth, Expert Rev. Mol. Diagn., 2015, 15, 23.

7. P. Jansen van Vuren, A. Grobbelaar, N. Storm, O. Conteh, K. Konneh, A. Kamara, I. Sanne, and J. T. Paweska, J. Clin. Microbiol., 2016, 54, 359.

8. H. Salimnia, M. R. Fairfax, P. R. Lephart, P. Schreckenberger, S. M. DesJarlais, J. K. Johnson, G. Robinson, K. C. Carroll, A. Greer, M. Morgan, R. Chan, M. Loeffelholz, F. ValenciaShelton, S. Jenkins, A. N. Schuetz, J. A. Daly, T. Barney, A. Hemmert, and K. J. Kanack, J. Clin. Microbiol., 2016, 54, 687.

9. M. Uehara, M. Hanamura, K. Yamada, A. Yamaguchi, T. Murayama, Y. Saito, K. Idegami, and T. Honda, Anal Sci., 2016, 32, 371 .

10. M. Okochi, H. Tsuchiya, F. Kumazawa, M. Shikida, and H. Honda, J. Biosci. Bioeng., 2010, 109, 193.

11. C. H. Chiou, D. J. Shin, Y. Zhang, and T. H. Wang, Biosens. Bioelectron., 2013, 50, 91.

12. H. Bordelon, P. K. Russ, D. W. Wright, and F. R. Haselton, PLoS One, 2013, 8, e68369.

13. S. A. Thatcher, Clin. Chem., 2015, 61, 89.

14. M. Uehara, K. Matsuda, M. Sugano, and T. Honda, J. Vet. Med. Sci., 2014, 76, 477.

15. World Health Organization, " $C D C$ Protocol of Real-time RT-PCR for Swine Influenza (H1N1)”, 2009, Geneva, World Health Organization, Switzerland, http://www.who.int/ csr/resources/publications/swineflu/CDCrealtimeRTP CRprotocol_20090428.pdf. 